\title{
Multiple Peaks in the Angular and Field Dependence of the Critical Currents in $\mathrm{Nb}-47 \mathrm{wt} \% \mathrm{Ti} / \mathrm{Cu}$ Multilayers
}

\author{
E. Kadyrov, L. D. Cooley, A. Gurevich, P. J. Lee and D. C. Larbalestier* \\ Applied Superconductivity Center, 1500 Engineering Dr., Madison, WI 53706
}

\begin{abstract}
The effect of $\mathrm{Nb}$-Ti layer thickness $\mathrm{d}_{s}$ on the angular and field dependencies of $\mathrm{J}_{\mathrm{c}}$ in $\mathrm{Nb}-47 \mathrm{wt}$.\% $\mathrm{Ti} / \mathrm{Cu}$ multilayers was investigated. $J_{c}$ exhibits several peaks in the field and angular dependencies for superconductor and pin layer thickness comparable to the coherence and proximity lengths. The shape of the $J_{c}(B)$ curve strongly depends on the thickness of $\mathrm{Nb}$-Ti layer. A single peak is present for $d_{s}>10 \mathrm{~nm}$ and multiple peaks (two and three) are observed in a sample with $d_{s}:=10 \mathrm{~nm}$. The low field peak is temperature independent, while the high-field peaks have a pronounced temperature dependence. We suggest that a crossover between dominant pinning mechanisms and orientational transitions in the vortex lattice is responsible for the observed behavior of $J_{c}(B)$.
\end{abstract}

\section{INTRODUCTION}

The main source of interest in superconducting artificial layered structures has been centered around the possibility of achieving higher critical current densities $\mathrm{J}_{\mathrm{c}}$. Superconductornormal metal-superconductor (SNS) multilayer structures are of special interest because of their resemblance to wires with artificial pinning centers $[1,2]$ and possibility of precise control of their microstructure on a nanometer scale. The superconducting order parameter is strongly modulated in these structures and consequently the normal metal layers act as effective vortex pinning centers. An additional advantage of low- $T_{c}$ multilayers as model structures is the long coherence length $\xi$ which is equal to $5.2 \mathrm{~nm}$ in $\mathrm{Nb}-\mathrm{Ti}$ at $4.2 \mathrm{~K}$, which makes accessible several regimes of flux pinning for relatively thick $\mathrm{N}$ layers. A number of reports dealing with the experimental study of $J_{c}$ in layered artificial superlattices have been published. Critical current measurements have been made on $\mathrm{Nb}$-Ta multilayers for both perpendicular and parallel applied field $b=B / B_{c 2}$, which indicated a direct summation of elementary core pinning forces. Measurements of critical currents of different multilayers in parallel fields revealed peaks in $\mathrm{J}_{\mathrm{c}}(\mathrm{B})$ which results in a non-monotonic dependence of $J_{c}(B)[4-7,11]$. Multiple peaks in $J_{c}(B)$ observed in Ref. [5], were attributed to the matching of the matching of the vortex lattice to the periodic multilayer structure. Reviews of experimental studies of flux pinning in multilayers are given in Ref. [6,7]. Since the increase of $J_{c}$ in the peaks can reach $100 \%$ at high fields [5], understanding the nature of this effect and the ability to control it are of great practical importance. New features in the angular dependence of $J_{c}$ occur in fields

Manuscript received August 27, 1996.

Research supported by the US Department of Energy, High Energy Physics Division, contracts DE-FG02-91ER40643 and DE-FG02-96ER40961.

*Also the Department of Materials Science and Engineering. inclined with respect to the layers, due to orientational transi tions in the vortex structure.

In order to elucidate the pinning mechanism for the multilayered systems and also to explore the possibility of practical applications, we have investigated the field and angular dependencies of $\mathrm{J}_{\mathrm{c}}$ of the $\mathrm{Nb}-47 \mathrm{wt} \% \mathrm{Ti} / \mathrm{Cu}$ multilayers with various $\mathrm{Nb}-\mathrm{Ti}$ layer thickness $10 \mathrm{~nm}<\mathrm{d}_{\mathrm{s}}<100 \mathrm{~nm}$. We have found that both the magnitude and the shape of the peaks in $J_{c}(B)$ strongly depend on $d_{s}$. The peaks are absent in the samples with large $d_{s}>100 \mathrm{~nm}$ and become more pronounced as $d_{s}$ is decreased. For the sample with $d_{s}=d_{n}=10 \mathrm{~nm}$, we observed multiple peaks in $J_{c}(B)$. The peaks in $J_{c}(B)$ are present in a broad range of field orientations $20^{\circ}<\theta<160^{\circ}$, where $\theta$ is the angle between $H$ and the normal to the layers. We also observed that the low field peak in $\mathrm{J}_{\mathrm{c}}(\mathrm{B})$ is temperature independent, while the high field peaks shift to higher fields as the temperature is decreased. We discuss the observed behavior of $J_{c}(B)$ in terms of crossover between different pinning mechanisms and vortex lattice orientations.

\section{EXPERIMENTAL}

We used the two-gun dc-magnetron sputtering to deposit $\mathrm{Nb}-47 \mathrm{wt} . \% \mathrm{Ti}$ and $\mathrm{Cu}$ layers onto sapphire substrates held at room temperature as described in [8]. Samples were fabricated with a copper layer thickness $d_{n}=10 \mathrm{~nm}$ and different $\mathrm{Nb}$-Ti layer thickness, $\mathrm{d}_{\mathrm{s}}=10,30$ and $100 \mathrm{~nm}$. The total sample thickness was equal to $1 \mu \mathrm{m}$ for all multilayers. Then 50 $\mu \mathrm{m}$ wide by $3 \mathrm{~mm}$ long bridges were patterned for the transport measurements.

Some of the multilayers were studied by transmission electron microscopy (TEM). An example of a multilayer TEM image is shown in Fig.1.

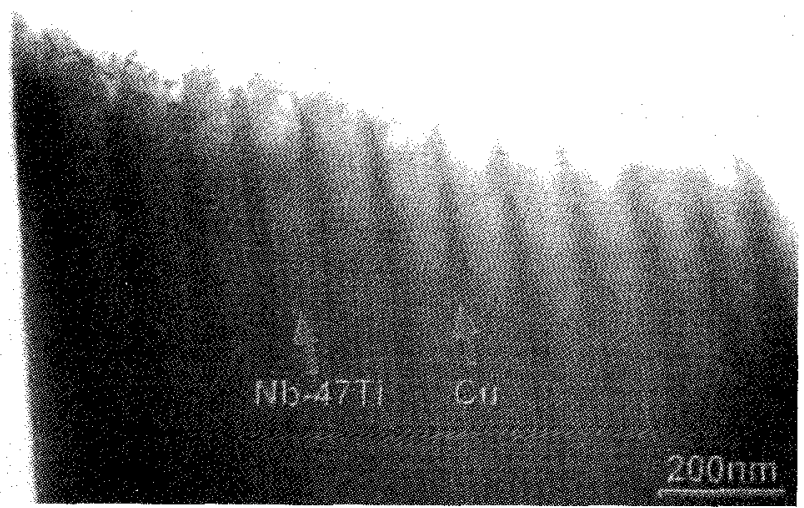

Fig.1. TEM image of multilayer with $d_{s}=70 \mathrm{~nm}$ and $d_{n}=30 \mathrm{~nm}$. 
$\mathrm{J}_{\mathrm{c}}$ was measured by the four probe method using a criterion of $1 \mu \mathrm{V} / \mathrm{cm}$. The magnetic field $0<\mu_{0} \mathrm{H}<12 \mathrm{~T}$ was always perpendicular to the transport current flowing along the layers. The angle $\theta$ between the external field $B$ and the normal to layers was varied with an angular resolution of $0.015^{\circ}$. Critical temperature $T_{c}$ and the upper critical field $B_{c 2}$ were obtained by measuring the sample resistivity $R(T, B)$ using the criterion $\mathrm{R}=0.001 \% \mathrm{R}_{\mathrm{n}}$, where $\mathrm{R}_{\mathrm{n}}$ is the normal state resistance. Critical temperatures were $8.22 \mathrm{~K}, 7.49 \mathrm{~K}$ and $6.52 \mathrm{~K}$ for $d_{s}=100,30$ and $10 \mathrm{~nm}$, respectively. We observed a nonmonotonic dependence of $\mathrm{J}_{\mathrm{c}}$ on $\mathrm{d}_{\mathrm{s}}$ in parallel field with maximum at $d_{s}=30 \mathrm{~nm}$ due to competition between magnetic pinning and proximity effect suppression of $\mathrm{T}_{\mathrm{c}}[8]$.

\section{RESULTS}

\section{A. Angular dependence of $J_{C}$}

Fig. 2 shows $J_{c}(\theta)$ curves measured at $B=1 T, T=4.2 \mathrm{~K}$ for multilayers with $d_{s}=100 \mathrm{~nm}$ (a) and $d_{s}=30 \mathrm{~nm}(b) . J_{c}(\theta)$ exhibits a sharp peak at $\theta=90^{\circ}$ [9]. It is also seen that both the shape of $\mathrm{J}_{c}(\theta)$ and the magnitude of $\mathrm{J}_{\mathrm{c}}$ in a parallel field strongly depends on $\mathrm{d}_{s}$. For instance, the decrease of $\mathrm{d}_{s}$ results in narrowing of the parallel field peak in $J_{c}(\theta)$ which can be described by the relation $J_{c}(\theta) \sim 1 /(\cos \theta)^{t}$, where exponent $t$ depend on $\mathrm{d}_{s}$. For the angles $1 \theta-90^{\circ} \mid<5^{\circ}, \mathrm{t}=1 / 2$ for $\mathrm{d}_{\mathrm{s}}=30$ $\mathrm{nm}$ and $\mathrm{t}=0.72$ for $\mathrm{d}_{\mathrm{s}}=100 \mathrm{~nm}$. In addition, the width and the shape of the peak in $J_{c}(\theta)$ strongly depend on B. For $d_{s}=$ $100 \mathrm{~nm}, \mathrm{~J}_{\mathrm{c}}(\mathrm{B})$ monotonically decreases with $\mathrm{B}$ for $30^{\circ}<\theta<$ $150^{\circ}$. By contrast, for $d_{s}=30 \mathrm{~nm}, J_{c}(B)$ dependence becomes non-monotonic in a broad angular range $30^{\circ}<\theta<150^{\circ}$. Notice that the dependence $J_{c}(\theta)$ is quite complex and can exhibit both a "shoulder" structure for $\mathrm{d}_{\mathrm{s}}=30 \mathrm{~nm}$ and additional peaks at $\theta=150^{\circ}$ for $d_{s}=100 \mathrm{~nm}$.

\section{B. Field dependence of $J_{c}$}

The field dependence $\mathrm{J}_{\mathrm{c}}(\mathrm{B})$ was measured for $1.9 \mathrm{~K}<\mathrm{T}<$ $9 \mathrm{~K}$ and different $\theta$. When the field was aligned parallel to the layers, $J_{c}(B)$ monotonically decreases with $B$ for $d_{s}=100 \mathrm{~nm}$, however a non-monotonic dependence of $J_{c}(B)$ develops for smaller $d_{s}<70 \mathrm{~nm}$. The peaks in $J_{c}(B)$ become more pronounced as $d_{s}$ is increased. For $d_{s}=10 \mathrm{~nm}$, we observed multiple peaks $J_{c}(B)$ [8]. Fig. 3 shows $J_{c}\left(B, 75^{\circ}\right)$ measured at different temperatures $T$ for the samples with $d_{s}=30$ (a) and 10 $n m(b)$. For $d_{s}=30 \mathrm{~nm}$, the $J_{c}\left(B, 75^{\circ}\right)$ curves exhibit a characteristic peak around $B \approx 1.5-3 \mathrm{~T}$ with the peak position depending on $T$. For $d_{s}=10 \mathrm{~nm}$, a double peak in $J_{c}\left(B, 75^{\circ}\right)$ was observed. An interesting feature of the $J_{\mathcal{c}}(B)$ dependence for $\mathrm{d}_{\mathrm{s}}=30 \mathrm{~nm}$ is a sharp increase of $\mathrm{J}_{\mathrm{c}}(\mathrm{B})$ around $\mathrm{B}=3 \mathrm{~T}$ at $1.9 \mathrm{~K}$. The increase of $\mathrm{T}$ smears out this peak which shifts to lower fields at higher temperature and disappears above $7 \mathrm{~K}$. For the sample with $d_{s}=10 \mathrm{~nm}$, the dependence $J_{c}(B)$ is more complex due to the appearance of multiple peaks. The shape of $\mathrm{J}_{\mathrm{c}}(\mathrm{B})$ and magnitude of peaks depend on the angle $\theta$. for
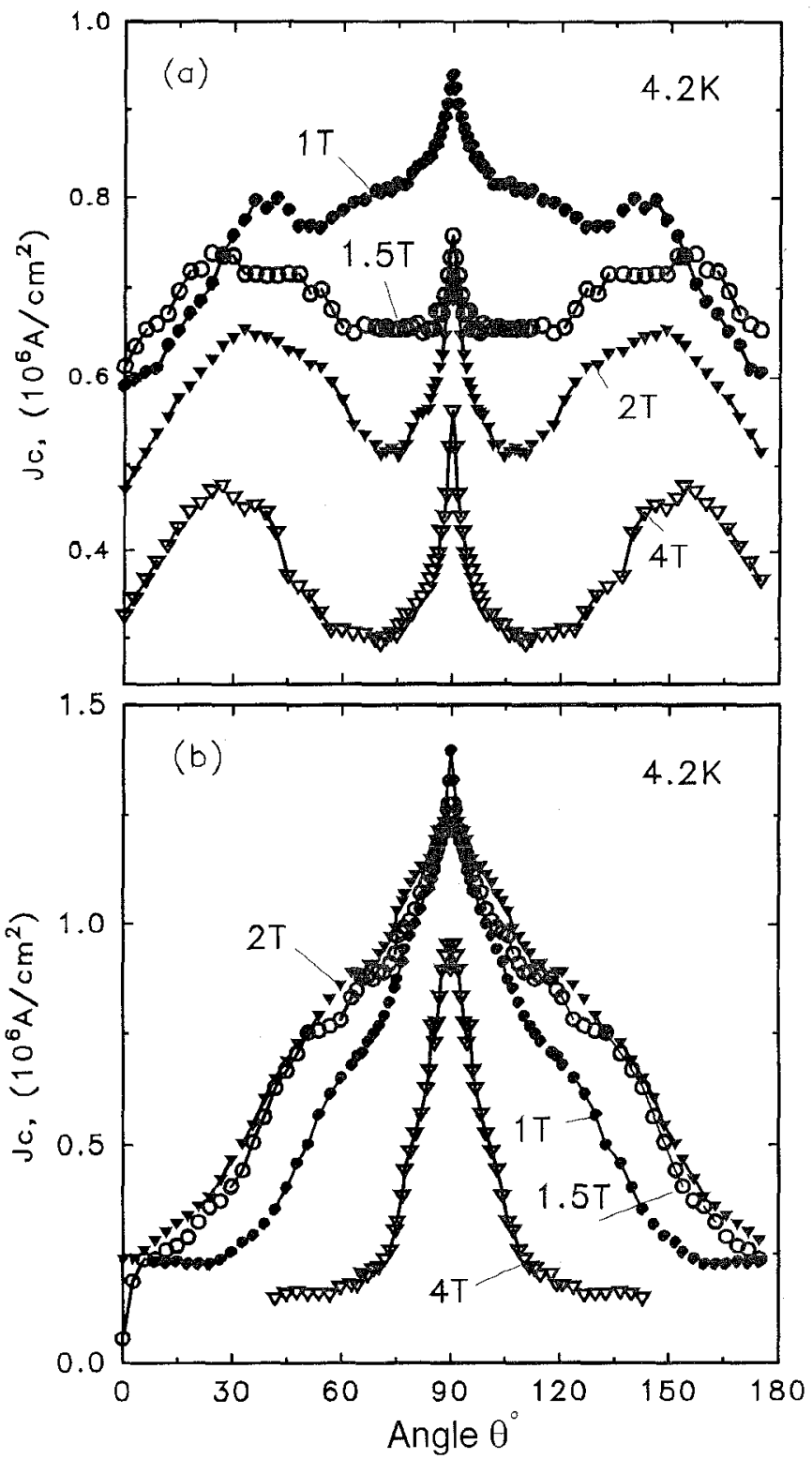

Fig. 2. Angular dependence $\mathrm{J}_{\mathrm{c}}(\theta)$ in $\mathrm{Nb}-\mathrm{Ti} / \mathrm{Cu}$ multilayer with (a) $\mathrm{d}_{\mathrm{s}}=100$ nm.; (b) $\mathrm{d}_{\mathrm{s}}=30 \mathrm{~nm}$.

example, three peaks are present at $\theta=75^{\circ}, 60^{\circ}$ and $45^{\circ}$ displays only two peaks, as shown in Fig. 4. The magnitude of the peaks at lower field is typically less than the magnitude of the higher field peaks. We observed no temperature dependence of the field position of the first peak and a noticeable temperature dependence of the position of the second peak. This is show $n$ on Fig. 3b where the field positions of the $\mathrm{J}_{\mathrm{c}}(\mathrm{B})$ peaks are indicated by the dashed lines. Similar dependencies (multiple peaks with different temperature dependence) were also observed in parallel field [8].

\section{DISCUSSION}

The complex non-monotonic field and angular dependencies of $\mathbf{J}_{\mathrm{c}}(\mathrm{B})$ observed in our multilayers reflect characteristic features of the interaction of the vortex lattice with the peri- 


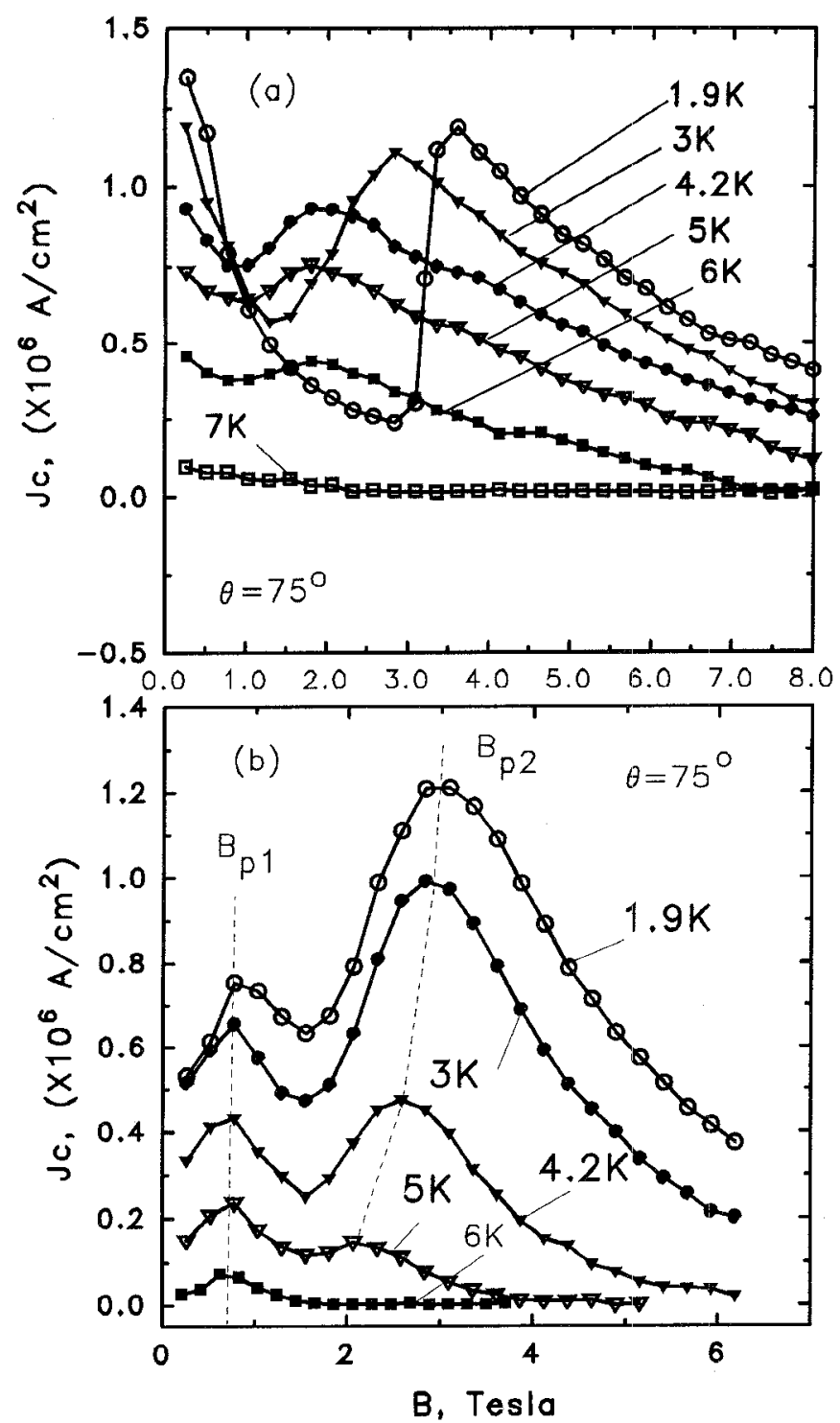

Fig. 3. $\mathrm{J}_{\mathrm{c}}(\mathrm{B})$ at $\theta=75^{\circ}$ for the sample with (a) $\mathrm{d}_{\mathrm{s}}=30 \mathrm{~nm}$; (b) $\mathrm{d}_{\mathrm{s}}=10 \mathrm{~nm}$.

odic multilayer structure. The string pinning by $\mathrm{N}$ layers tends to align vortices parallel to the layers, whereas the magnetic interaction tends to align vortices along the applied field B. A competition between these effects gives rise to a kinked vortex structure in the case of inclined fields B [9]. In addition, the discreteness of the vortex structure results in matching effects which manifest themselves in peaks in $\mathrm{J}_{c}(B)$ at fields $B_{n}$ for which the spacing between neighboring vortex rows becomes commensurate with the multilayer period $l=d_{n}+d_{s}$. In this case the matching fields $B_{n}=\varepsilon \phi_{0} \sqrt{ } 3 / 2 n^{2} 1^{2}$ weakly depends on $T$ only via the ratio $\varepsilon=\lambda_{\perp} / \lambda_{\|}$of the effective penetration depths perpendicular $\left(\lambda_{\perp}\right)$ and parallel $\left(\lambda_{\|}\right)$to the layers, where $n$ is integer. In this case multiple peaks in $J_{c}(B)$ should be seen at $B=B_{n}[5,10]$.

Another possibility for the peak effect in inclined fields can be due to crossover from an anisotropic Abrikosov lattice at low fields [11], to the kinked vortex structure at higher fields [13]. The kinked vortex lattice is more strongly pinned than

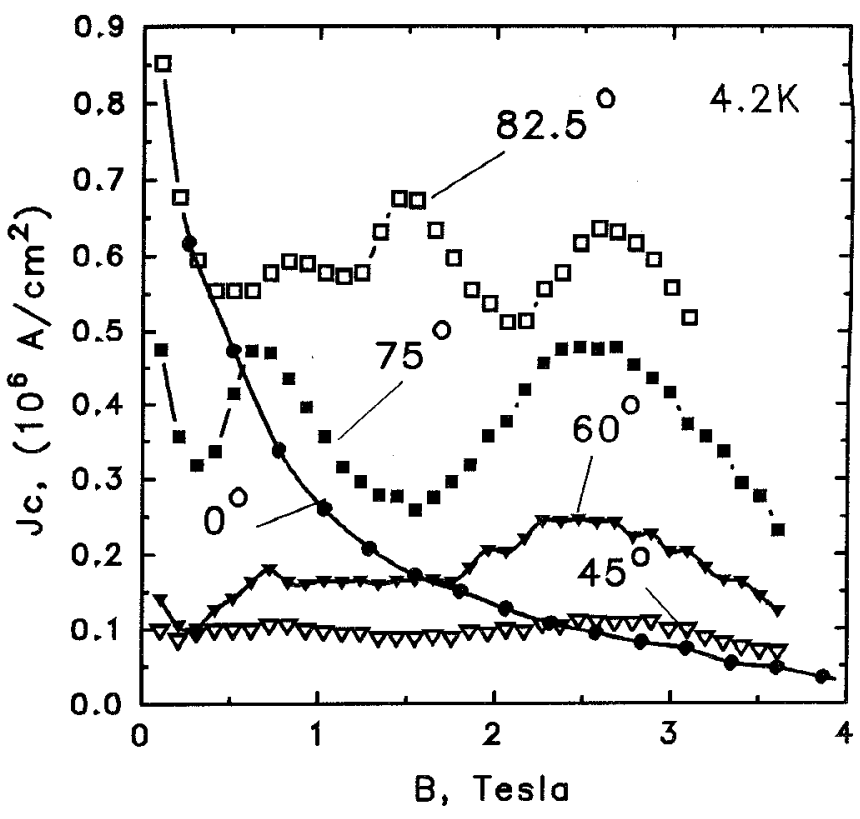

Fig. 4. $J_{c}(B)$ at $T=4.2 \mathrm{~K}$ for sample with $\mathrm{d}_{\mathrm{s}}=10 \mathrm{~nm}$.

the unkinked lattice and this produces a peak in $\mathrm{J}_{\mathrm{c}}$ near crossover. Nojima et al. [7] have suggested that the peak in $\mathbf{J}_{\mathrm{c}}$ may be due to the field induced decoupling of $\mathrm{Nb}$ layers above $\mathrm{B}$ $>\mathrm{B}_{\max }$ and corresponding increase in the elementary pinning force due to core pinning mechanism. Softening of the tilt modulus $\mathrm{c}_{44}$ and decrease in the rigidity of the vortex lattice relative to the intrinsic pinning force $f_{p}$ is suggested as a mechanism of the peak effect in [4]. Yet another mechanism of magnetic pinning is shown to be dominant in $\mathrm{Nb}-\mathrm{Ti} / \mathrm{Cu}$ multilayers [8] which also exhibit strong non-monotonic dependence of $\mathrm{J}_{\mathrm{c}}(\mathrm{B})$.

None of the above models is sufficient to explain the variety of the angular and field dependencies of $J_{c}(B)$ observed in our multilayers. For example, orientational transition in the vortex lattice or decoupling of the pin layer do not explain multiple peaks, while matching of the lattice periodicity to the multilayer period is inconsistent with the observed temperature dependence of the peaks. The multiple peak effect observed in our experiments may be due to combination of the following physical mechanisms.

At low field, vortices first penetrate the proximity coupled $\mathrm{N}$ pin layers and a non-monotonic dependence of the pinning force on $\mathrm{B}$ may result from a field-induced decoupling of $\mathrm{N}$ layers $[8,12]$. This model would seem to apply at low $\mathrm{B}$ when there are no fluxons in the $\mathrm{Nb}$-Ti layers. As a result, the first peak in $J_{c}(B)$ for $d_{s}=10 \mathrm{~nm}$ and $d_{s}=30 \mathrm{~nm}$ would be consistent with an increase of the pinning force of the $\mathrm{Cu}$ layers due to the suppression of their proximity coupling. Another possible reason for the first peak is matching of periodic lattice to the periodic pinning potential, which results in temperature independent peaks in $d_{s}=10 \mathrm{~nm}$. However strong pinning characteristics of our samples effectively destroys long range order in the vortex lattice which is necessary for matching effects. As the field further increases, vortices start to penetrate into the superconducting layers, which results in the de- 
crease of $J_{c}(B)$ above $B_{m}$. A rough estimate of $B_{m}$ could be made using the formula for the lower critical field $\mathrm{B}_{\mathrm{cl}}=$ $\left(2 \phi_{0} / \pi d_{s}^{2}\right) \ln \left(d_{s} / \xi\right)$ for a film with thickness $d_{s}<\lambda$. For the sample with $\mathrm{d}_{\mathrm{s}}=10 \mathrm{~nm}$, we have $\mathrm{B}_{\mathrm{c} 1} \leftrightharpoons 8.1 \mathrm{~T}$. The effective thickness of the superconducting must however be modified in the present case because of the significant supercurrent which can flow through $\mathrm{N}$ layer due to proximity effect. In this case $B_{c 1}$ - $6 \mathrm{~T}$ seems to be a reasonable value, based on multilayer wavelength being $20 \mathrm{~nm}$. The corresponding value of $B_{m}$ for $d_{s}=30 \mathrm{~nm}$ is about $2 \mathrm{~T}$. This means that effective pinning by the copper layer persists up to at least a few tesla, until fluxons begin to penetrate the $\mathrm{Nb}$-Ti layers.

At $\mathrm{B}>\mathrm{B}_{\mathrm{m}}$ vortices penetrating superconducting layers reduce $J_{c}(B)$, because the larger number of fluxons generate a greater Lorentz force against the same number of pinning interactions. This results in a decrease of $J_{c}(B)$ at $B>B_{m}$, as is seen in Fig. 3. However, if the coupling strength of the copper layers is decreased by the field sufficiently, the magnetic interaction of the fluxons in the $\mathrm{Nb}-\mathrm{Ti}$ layer with the $\mathrm{Nb}-\mathrm{Ti} / \mathrm{Cu}$ interface will then provide a strong magnetic pinning first considered by Shmidt for thin films[13].

This situation was analyzed recently by Zeise et al. [14], who found that the interface pinning force may be strong enough to produce currents similar in magnitude to those predicted by other flux-pinning models. The second peak in $J_{c}(B)$ for $d_{s}=10 \mathrm{~nm}$ then can be explained by increasing magnetic pinning force due to interface surface barrier.

In conclusion we have studied the effect of superconductor layer thickness variation on the field and angular dependence of $\mathrm{J}_{\mathrm{c}}$ in $\mathrm{Nb}-47 \mathrm{wt} \% \mathrm{Ti} / \mathrm{Cu}$ multilayers. A transition from monotonic to non-monotonic $J_{c}(B)$ dependence is found in a broad angular range $20^{\circ}<\theta<160^{\circ}$ for $\mathrm{d}_{\mathrm{s}}<100 \mathrm{~nm}$. The pres- ence of multiple peaks with different temperature dependence suggests a crossover from a core to magnetic pinning mechanism at higher field.

\section{REFERENCES}

[1] L. R. Motowidlo, B. A. Zeitlin, M. S. Walker and P. Haldar, Appl Phys Lett., vol . 61, pp. 991-993, 1992.

[2] K. Matsumoto, H. Takewasi, Y. Tanaka, O. Miura, K. Yamafuji, K. Funaki, M. Iwakuna and T. Matsushita, Appl Phys Lett., vol. 64, pp. 115-120,1994.

[3] P. R. Broussard, T. H. Geballe, Phys. Rev. B, vol. 37(1), pp. 68 75, 1988.

[4] J. McCambridge, N. D. Rizzo, S. T. Hess, Jian Q. Wang, X. S. Ling and D. Prober, IEEE Trans. Appl. Superc., vol. 5, pp. 1697-1701, 1995.

[5] H. Raffy and J. C. Renard, Solid State Comm., vol. 11, 1679, 1972.

[6] A. N. Lykov, Physica C, vol. 218, pp. 485-487, 1993.

[7] T. Nojima, M. Kinoshita, S. Nakano, Y. Kuwasawa, Physica $C$, vol. 206, pp. 387-392, 1993.

[8] E. Kadyrov, A. Gurevich and D. C. Larbalestier, Appl. Phys. Lett., vol. 68(11), pp. 1567-1570, 1996.

[9] M. Tachiki and S. Takahashi, Phys. Rev.B, vol. 47, pp. 6618 6626, 1996.

[10] S. Ami and K. Maki, Prog. Theor. Phys., vol. 53, pp.1 -21, 1975.

[11] P. Koorevaar, W. Maj, P. H. Kes and J. Aarts, Phys. Rev. B, vol. 47, pp. 934-942, 1993.

[12] L. D. Cooley, P. J. Lee and D. C. Larbalestier, Phys. Rev. B, vol.53(10), pp. 6618-6626, 1996.

[13] V.V. Shmidt, Soviet Phys. JETP, vol. 30(6), pp. 1137-1143, 1970.

[14] M. Ziese, P. Esquinazi, P. Wagner, H. Adrian, S. Brongersma and R. Griessen, Phys. Rev. B, vol.(13), pp. 8658-8670, 1996. 